\title{
Intraocular lens optic capture in pediatric cataract surgery
}

\begin{abstract}
Pediatric cataract surgery differs from adult cataract surgery in the very high incidence of posterior capsule opacification and secondary membrane formation. Specialized techniques need to be performed at the time of surgery to minimize this complication. This article reviews three techniques of intraocular lens optic capture to decrease the incidence of secondary membranes and capsule opacification.
\end{abstract}

Keywords: pediatric cataract surgery, posterior capsule opacification, secondary membranes, intraocular lens capture, posterior capsulorhexis with optic capture, double optic capture with capsular bag fusion
Volume I Issue 2 - 2014

\section{Brian M DeBroff}

Department of Ophthalmology and Visual Science, Yale University, School of Medicine, USA

\begin{abstract}
Correspondence: : Brian M DeBroff, Department of Ophthalmology and Visual Science, Yale University, School of Medicine, New Haven, CT, USA, Tel 066|4-203-375-5819, Emailbmdmd@aol.com
\end{abstract}

Received: August 27, 2014 | Published: September II, 2014

\begin{abstract}
Abbreviations: IOL, intraocular lens; PCCC, posterior continuous curvilinear capsulorhexis
\end{abstract}

\section{Introduction}

A primary concern in pediatric intraocular lens (IOL) implantation surgery is the common occurrence of posterior capsular opacification. Lens epithelial cells proliferate and undergo myofibroblastic transformation. ${ }^{1}$ Secondary membranes result that use a scaffold of the anterior vitreous face or the anterior/posterior capsules., ${ }^{2,3}$ Capturing of the IOL through both anterior and posterior capsulotomy openings allows fusion of the capsular bag and thus prevention of lens epithelial migration to the central visual axis. This paper describes three methods of performing optic capture in children to achieve fusion of the anterior and posterior capsule leaflets.

\section{Posterior capsulorhexis with optic capture}

Posterior capsulorhexis with optic capture was originally described by Gimbel \& DeBroff $f^{4}$ to maintain a clear visual axis after pediatric cataract surgery. This technique involves capturing the IOL optic through a primary posterior continuous curvilinear capsulorhexis (PCCC), while the haptics remain within the capsular bag (Figure 1). Studies have demonstrated this to be a valuable technique to prevent secondary cataract formation and to maintain excellent IOL centration for children under the age of 6 in whom a primary posterior capsulotomy is advocated..$^{5-12}$

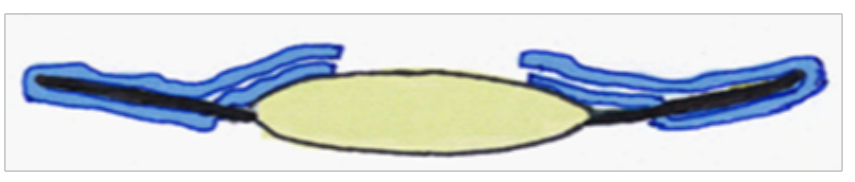

Figure I The appearance of an IOL after posterior capsulorhexis with optic capture.This same figure also depicts the final result of posterior capsulotomy with optic capture. The IOL haptics remain in the capsular bag.

A PCCC is performed after cataract removal either before or after IOL placement into the capsular bag. An advantage of performing the PCCC after placement of the IOL into the capsular bag is the ability to discontinue the PCCC with the IOL already safely positioned in the bag. Others prefer to perform the posterior capsulorhexis before IOL implantation. ${ }^{13}$ Posterior capture of the IOL optic is achieved by gentle pressure of one half of the IOL optic followed by slow and gentle pressure of the second half of the optic through the capsulorhexis opening. An anterior vitrectomy should be performed if vitreous herniates through the posterior capsulorhexis opening. Some studies have demonstrated that an anterior vitrectomy done routinely in conjunction with PCCC with optic capture may be beneficial, especially in children under the age of 5 years ${ }^{14-16}$ and as a method to improve low-contrast sensitivity. ${ }^{13} \mathrm{~A}$ prospective randomized study involving 34 eyes by Raina et al. ${ }^{17}$ however, found that PCCC with optic capture prevents secondary opacification of the visual axis in the absence of an anterior vitrectomy. It has been postulated that even in the absence of lens epithelial cells migration, vitreous opacification may occur as a result of a primary response of the anterior vitreous face coming contact with the IOL edge, and this may be an additional reason to consider performing a routine anterior vitrectomy with optic capture. ${ }^{13}$

In summary the technique of Posterior Capsulorhexis with Optic Capture creates an opposition of the anterior and posterior capsule leaflets anterior to the IOL and thus enables an effective sealing of the capsular bag that prevents migration of lens epithelial cells posterior to the IOL. This technique has been demonstrated to help keep the optical axis clear while maintaining excellent support and centration of the implant. The disadvantages of this technique are that it is technically very challenging especially in younger children where the eye is small and the anterior and posterior capsules are very thin and elastic in nature. If an attempt is made to capture the IOL through too small a posterior capsulorhexis, loss of bag integrity can occur, which may limit the ability to place an IOL. In addition if the posterior capuslorhexis is too large, capture will be impossible.

\section{Double optic capture with capsular bag fusion}

In double optic capture with capsular bag fusion the IOL haptics are placed in the ciliary sulcus and the IOL optic is captured through both the anterior continuous curvilinear capsulorhexis and the posterior capsulotomy (Figure 2). ${ }^{18}$ This technique involves performing an anterior capsulorhexis opening approximately one millimeter in diameter smaller than the IOL optic. A matching posterior capsulotomy opening is then performed using the vitrectomy hand piece. Even if the anterior capsulorhexis opening is larger than the IOL diameter, the posterior capsulotomy size can be adjusted so that it is smaller than the IOL optic diameter and thus still allow effective capture. The posterior capsule is elastic enough to withstand the capture process 
without tearing even though a vitrectomy hand piece was used to create the opening versus a continuous curvilinear capsulorhexis. By eliminating the need to perform a posterior capsulorhexis, the most demanding, difficult, and highest potential for complication aspect of the PCCC with optic capture is eliminated.

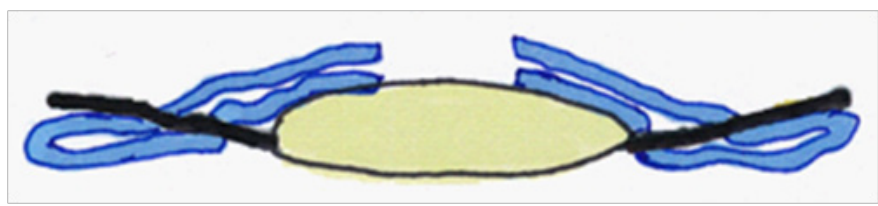

Figure 2 The appearance of the IOL after double optic capture with capsular bag fusion. The IOL haptic remain in the ciliary sulcus.

Double optic capture with capsular bag fusion allows fusion of the anterior and posterior leaflets of the capsular bag for 360 degrees. The optic capture helps to achieve a well-centered IOL that is resistant to movement and chafing of uveal tissue even with the IOL haptics in the sulcus. The major advantage of double optic capture technique with capsular bag fusion is the ease of performing the procedure even in young children (no posterior capsulorhexis required), the stability of the IOL (due to the capture process) and minimization of secondary membranes (because of complete fusion of the capsule leaflets). The main disadvantages of sulcus IOL placement (uveal rub and decentration) are prevented by the capture technique.

\section{Posterior capsulotomy with optic capture}

The technique of double optic capture with capsular bag fusion demonstrated that capture of the IOL optic through a posterior capsule opening made with an anterior vitrectomy is just as resistant to tears as capture through a capsulorhexis opening. Thus, a new technique, posterior capsulotomy with optic capture, was developed in which the surgeon performs an anterior CCC, removal of the cataract, and a posterior capsulotomy and limited anterior vitrectomy using an anterior vitrectomy hand piece. The IOL is then placed in the capsular bag and the optic captured through the posterior capsulotomy opening (Figure 1). The advantage of this technique is that the difficult step of posterior continuous curvilinear capsulorhexis has been eliminated. Also, the posterior capsulotomy size can be more accurately achieved using the anterior vitrectomy hand piece. This technique is still in experimental use, but initial results have been encouraging. The posterior capsulotomy opening can also be made using the femtosecond laser; however, this requires an additional docking and laser step after cataract removal and still requires an anterior vitrectomy to be subsequently performed. In summary, there are multiple techniques to allow placement of an IOL in children which can help prevent capsular opacification and posterior membranes. This is achieved by capturing the IOL optic allowing the anterior and posterior capsule leaflets to fuse and prevent migration and proliferation of lens epithelial cells. The ideal technique may require additional controlled studies performed by multiple surgeons.

\section{Acknowledgments}

None.

\section{Conflicts of interest}

Author declares that there is no conflict of interest.

\section{References}

1. McDonnell PJ, Zarbin MA, Green WR. Posterior capsule opacification in pseudophakic eyes. Ophthalmology. 1983;90(12):1548-1553.
2. Cobo LM, Ohsawa E, Chandler D, et al. Pathogenesis of capsular opacification after extracapsular cataract extraction. An animal model. Ophthalmology. 1984;91(7):857-863.

3. Nishi O. Fibrinous membrane formation on the posterior chamber lens during the early postoperative period. J Cataract Refract Surg. 1988;14(1):73-77.

4. Gimbel HV, DeBroff BM. Posterior capsulorhexis with optic capture: Maintaining a clear visual axis after pediatric cataract surgery. $J$ Cataract Refract Surg. 1994;20(6):658-664.

5. Gimbel HV, DeBroff BM. Surgical management of pediatric cataracts McGill University, Toronto. Ophthalmology. 1994;6(1):2-6.

6. Gimbel HV. Posterior capsulorhexis with optic capture in pediatric cataract and intraocular lens surgery. Ophthalmology. 1996;103(11):1871-1875.

7. Gimbel HV. Posterior continuous curvilinear capsulorhexis and optic capture of the intraocular lens to prevent secondary opacification in pediatric cataract surgery. J Cataract Refract Surg. 1997;23(Suppl 1):652-656.

8. Metori Y, Kageyama T, Aramaki T, et al. Pediatric cataract surgery with posterior capsulorhexis and optic capture of the intraocular lens. Nihon Ganka Gakkai Zasshi. 2000;104(2):91-96.

9. Gimbel HV, DeBroff BM. Management of lens implant and posterior capsule with respect to prevention of secondary cataract. Operative Tech Cataract Refract Surg. 1998;1:185-190.

10. Gimbel HV, Dahan E, DeBroff BM. Surgical management of pediatric cataracts. In: Steinert RF, editor. Cataract Surgery: Technique, Complication, \& Management. PA, Saunders, USA; 1995. p. 247-267.

11. Vasavada AR, Trivedi RH. Role of optic capture in congenital cataract and intraocular lens surgery in children. $J$ Cataract Refract Surg. 2000;26(6):824-831.

12. Kohnen T, Pena-Cuesta R, Koch DD. Secondary cataract formation following pediatric intraocular lens implantation: 6-month results. Ger J Ophthalmol. 1996;5(3):171-175.

13. Vasavada AR, Trivedi RH, Singh R. Necessity of vitrectomy when optic capture is performed in children older than 5 years. $J$ Cataract Refract Surg. 2001;27(8):1185-1193.

14. Koch DD, Kohnen T. A retrospective comparison of techniques to prevent secondary cataract formation following posterior chamber intraocular lens implantation in infants and children. Trans Am Ophthalmol Soc. 1997;95:351-360.

15. Koch DD, Kohnen T. Retrospective comparison of techniques to prevent secondary cataract formation after posterior chamber intraocular lens implantation in infants and children. J Cataract Refract Surg. 1997;23(Suppl 1):657-663.

16. Vasavada A, Desai J. Primary posterior capsulorhexis with and without anterior vitrectomy in congenital cataracts. J Cataract Refract Surg. 1997;23(Suppl 1):645-651.

17. Raina UK, Gupta V, Arora R, et al. Posterior continuous curvilinear capsulorhexis with and without optic capture of the posterior chamber intraocular lens in the absence of vitrectomy. $J$ Pediatr Ophthalmol Strabismus. 2002;39(5):278-287.

18. DeBroff BM, Nihalani BR. Double optic capture with capsular bag fusion: A new technique for pediatric intraocular lens implantation. Techniques in Opthalm. 2008;6(2):31-34. 